\title{
Retraction
}

\section{Retracted: Experimental Investigation on Hydraulic Fractures in the Layered Shale Formation}

\author{
Geofluids \\ Received 10 June 2020; Accepted 10 June 2020; Published 2 September 2020 \\ Copyright (c) 2020 Geofluids. This is an open access article distributed under the Creative Commons Attribution License, which \\ permits unrestricted use, distribution, and reproduction in any medium, provided the original work is properly cited.
}

Geofluids and the authors have retracted the article titled "Experimental Investigation on Hydraulic Fractures in the Layered Shale Formation" [1].

The authors have identified errors in the acoustic emission data which were highlighted during a repeat of the experiment. As these errors invalidate the conclusions made and cannot be corrected, the article is being retracted at the request of the authors and with the agreement of the editorial board.

\section{References}

[1] J. Wang, Y. Guo, K. Zhang, G. Ren, and J. Ni, "Experimental Investigation on Hydraulic Fractures in the Layered Shale Formation," Geofluids, vol. 2019, Article ID 4621038, 14 pages, 2019. 


\title{
Experimental Investigation on Hydraulic Fractures in the Layered Shale Formation
}

\author{
Jingyin Wang, ${ }^{1,2,3}$ Ying Guo $\mathbb{D}^{4}{ }^{4}$ Kaixun Zhang, ${ }^{5}$ Guangying Ren, ${ }^{4}$ and Jinlong $\mathrm{Ni}^{2}$ \\ ${ }^{1}$ Key Laboratory of Unconventional Oil \& Gas Development (China University of Petroleum (East China)), Ministry of Education, \\ Qingdao 266580, China \\ ${ }^{2}$ Shandong Provincial Key Laboratory of Depositional Mineralization \& Sedimentary Mineral, Shandong University of Science \\ and Technology, Qingdao, 266590 Shandong, China \\ ${ }^{3}$ School of Petroleum Engineering, China University of Petroleum (East China), Qingdao 266580, China \\ ${ }^{4}$ Institute of Geology and Paleontology, Linyi University, Linyi, 276000 Shandong, China \\ ${ }^{5}$ Institute of Geomechanics, Chinese Academy of Geological Sciences, Beijing 100081, China
}

Correspondence should be addressed to Ying Guo; guoying@lyu.edu.cn

Received 28 July 2019; Revised 9 October 2019; Accepted 25 October 2019; Published 29 November 2019

Academic Editor: Andrea Brogi

Copyright (c) 2019 Jingyin Wang et al. This is an open access article distributed under the Creative Commons Attribution License, which permits unrestricted use, distribution, and reproduction in any medium, provided the original work is properly cited.

\begin{abstract}
Multistage fracturing of horizontal wells to form a complex fracture network is an essential technology in the exploitation of shale gas. Different from the conventional reservoirs, the mechanical characteristics of shale rock have significant heterogeneity due to the existence of beddings, which makes it difficult to predict the fracture geometry in the shale reservoir. Based on the laboratory experiments, the factors that affect fracture propagation were analyzed. The experimental results revealed that the hydraulic fracture would cross the beddings under the high vertical stress difference, while it would propagate along with the bedding under the low vertical stress difference; besides, the low injection rate and viscosity of the fracturing fluid were beneficial to generate a complex fracture network. Under the high injection rate and viscosity, a planar fracture was created, while a nonplanar fracture was observed under the low injection rate and viscosity, and branch fracture was created. According to the acoustic emission events, the shear events were the main events that occurred during the hydraulic fracturing process, and the acoustic emission events could be adopted to describe the fracture network. Lastly, the supercritical carbon dioxide fracturing was more effective compared with the hydraulic fracturing because the fracture network was more complex.
\end{abstract}

\section{Introduction}

Hydraulic fracturing has become an essential technology in the exploration of shale reservoir. Because of the existence of bedding planes and natural fractures, it is difficult to predict the fracture geometry in shale reservoir [1-8]. However, it is of great importance to understand the fracture initiation and propagation mechanism in the vertical plane for estimating the stimulated reservoir volume.

The vertical heterogeneity of shale rock mechanics has been recognized as an important factor to affect the fracture propagation in the vertical direction. Some researchers [9-13] have studied the factors which affected the vertical propagation of the hydraulic fracture, which included the in situ stress, fluid viscosity, fluid flow rate, Young's mod- ulus, Poisson's ratio, beddings, natural fractures, and fracture toughness. In addition, the fracture geometry was also affected by the interaction between hydraulic fractures and beddings or natural fractures [14-16]. Some researchers have studied the fracture geometry when the hydraulic fracture came across the beddings or natural fractures. It was found that different types of interactions between hydraulic fractures and natural fractures were observed where the hydraulic fractures crossed and arrested, as well as opened and dilated, the natural fractures [17-19]. Liu et al. [20] studied the fracture propagation in fractured formation. They found that the hydraulic fracture crossed the preexisting fracture under high horizontal differential stress. Based on the laboratory experiment, Hou et al. [21] and Zhou et al. [22] found that the preexisting 
fractures near the wellbore could dramatically decrease the initiation and propagation pressure. Lee et al. [23] found that the approach angle had a great effect on the fracture propagation path. The flow rate and viscosity were also important parameters that affect the fracture propagation. Based on the laboratory experiment, it was demonstrated that the hydraulic fracture would cross the natural fracture under the high flow rate and viscosity, while the hydraulic fracture would deviate to the natural fracture under the low flow rate and viscosity. The numerical simulation was also an effective method to investigate the fracture propagation in fractured formation. Based on the finite element method, Guo et al. [15] investigated the relationship between horizontal differential stress and fracture propagation path based on numerical simulation. They found that a large approaching angle was a comparatively detrimental factor that negatively influences fracture network generation. Keshavarzi and Mohammadi [24] found that the natural fracture would divert hydraulic fractures at a low angle of approach, while the hydraulic fracture would cross the natural fracture at a high angle of approach. Jin [25] discussed the relationship between breakdown pressure and approach angle [26].

From the literature review above, the fracture propagation mechanism in the vertical plane has not been understood. Most attention focused on the fracture propagation in the horizontal plane. In this paper, a true triaxial experiment was conducted to study the vertical propagation behavior of hydraulic fractures and analyze the multiple factors influencing the vertical propagation of hydraulic fractures followed the methods of Jin et al. $[25,26]$. In addition, the acoustic emission monitoring system was used to monitor the hydraulic fracturing experiment. This research enhanced the understanding fracture propagation mechanism in the vertical plane, which also provided a reference for estimating the stimulated reservoir volume.

\section{Experiment Description}

2.1. Experimental Equipment and Sample. The true triaxial hydraulic fracturing simulation experiments were conducted with large-size shale cores to understand the vertical propagation of hydraulic fractures under different reservoir mechanical conditions and operating conditions. The experimental apparatus includes a triaxial hydraulic fracturing test system, acoustic emission monitoring system, and IPT4106D CT scanning system. The triaxial hydraulic fracturing test system is mainly composed of the triaxial model, stress loading system, constant pressure pump, electrical controlling system, data collection system, and auxiliary device. In this experiment, the prepared shale blocks are positioned in between the pressurized pistons in a true triaxial machine; the pistons are able to simulate the in situ stress conditions. The pressure platens are provided with foursquare sheets to ensure equal pressure distribution. Between the cubic model block and the pressure platen, a thin Teflon sheet was inserted which was covered on all sides with Vaseline. Vaseline was used to prevent the generation of shear stress between the pressure platens and the model block. Loading stresses that mimic the in situ stress field were applied to the specimen by using the confining pressure system. The maximum fracturing fluid injection rate and injection pressure of the constant pressure pump are $60 \mathrm{~mL} / \mathrm{min}$ and $60 \mathrm{MPa}$, respectively.

In order to specify the location of rock failure, the acoustic emission monitoring system is used to monitor the hydraulic fracturing experiment. Acoustic emission is an experimental method for studying the internal state and mechanical properties of rocks by the elastic waves. During hydraulic fracturing, when the rock is damaged by the high pressure caused from the fluid, the stress concentration is formed around the original or newly formed fractures in the rock. The nonuniform distribution of local stress concentration areas results in radial elastic waves, which mainly include $\mathrm{P}$-wave and $\mathrm{S}$-wave. Then, the data acquisition system is used to receive and detect the acoustic signals, and the acoustic data is processed and calculated to obtain the specific location of the rock fracture. In the test, the number of acoustic emission is collected by a single channel, and the event of acoustic emission is judged according to the polarity of $\mathrm{P}$-wave touching. When the acoustic emission source is a pure dilatant tensile fracture, the medium around the source will be squeezed and the probe will be connected; a compression waveform with $\mathrm{P}$-wave touching polarity upward is received. When the source of acoustic emission is a pure compressive closed failure, the expansion of the surrounding medium produces compressive force on the source, and the probe receives a $\mathrm{P}$-wave-driven downward expansion waveform. When shear-type failure occurs in the acoustic emission source, the medium part around the acoustic emission source is compressed and partly expands. The probe receives waveforms that produce different polarities. Therefore, it is defined that when more than $70 \%$ of the probes detect compressed waves, the AE event is defined as a tensional failure; when the AE event is less than $30 \%$, it is defined as a closed failure; when the $\mathrm{AE}$ event is between $30 \%$ and $70 \%$, it is a shear failure.

CT scanning is an effective method to understand the internal fracture morphology of rock samples. Compared with the CT scanning results, the accuracy of acoustic emission location results can be verified, and the influence of the original fractures on the expansion of hydraulic fractures can also be analyzed. The IPT4106D testing system can provide different CT scanning modes for different requirements and also provide a DR transmission scanning mode. It consists of six subsystems: ray source subsystem, linear array detector subsystem, surface array detector subsystem, and scanning device (Figure 1).

Multistaged fracturing is often used in cased and perforation horizontal wells in shale reservoir. In the experiment, the size of the shale sample is $30 \times 30 \times 30 \mathrm{~cm}^{3}$. The vertical stress is perpendicular to the bedding planes, the maximum horizontal stress is along the perforation hole, and the minimum horizontal stress is parallel to the beddings. A deep counter bore with a length of $17 \mathrm{~cm}$ and a diameter of $5 \mathrm{~cm}$ is drilled parallel to the beddings. By using epoxy glue, a steel liquid injection tube with a length of $13 \mathrm{~cm}$ and a diameter of $3 \mathrm{~cm}$ was fixed, leaving an open hole section with a length 


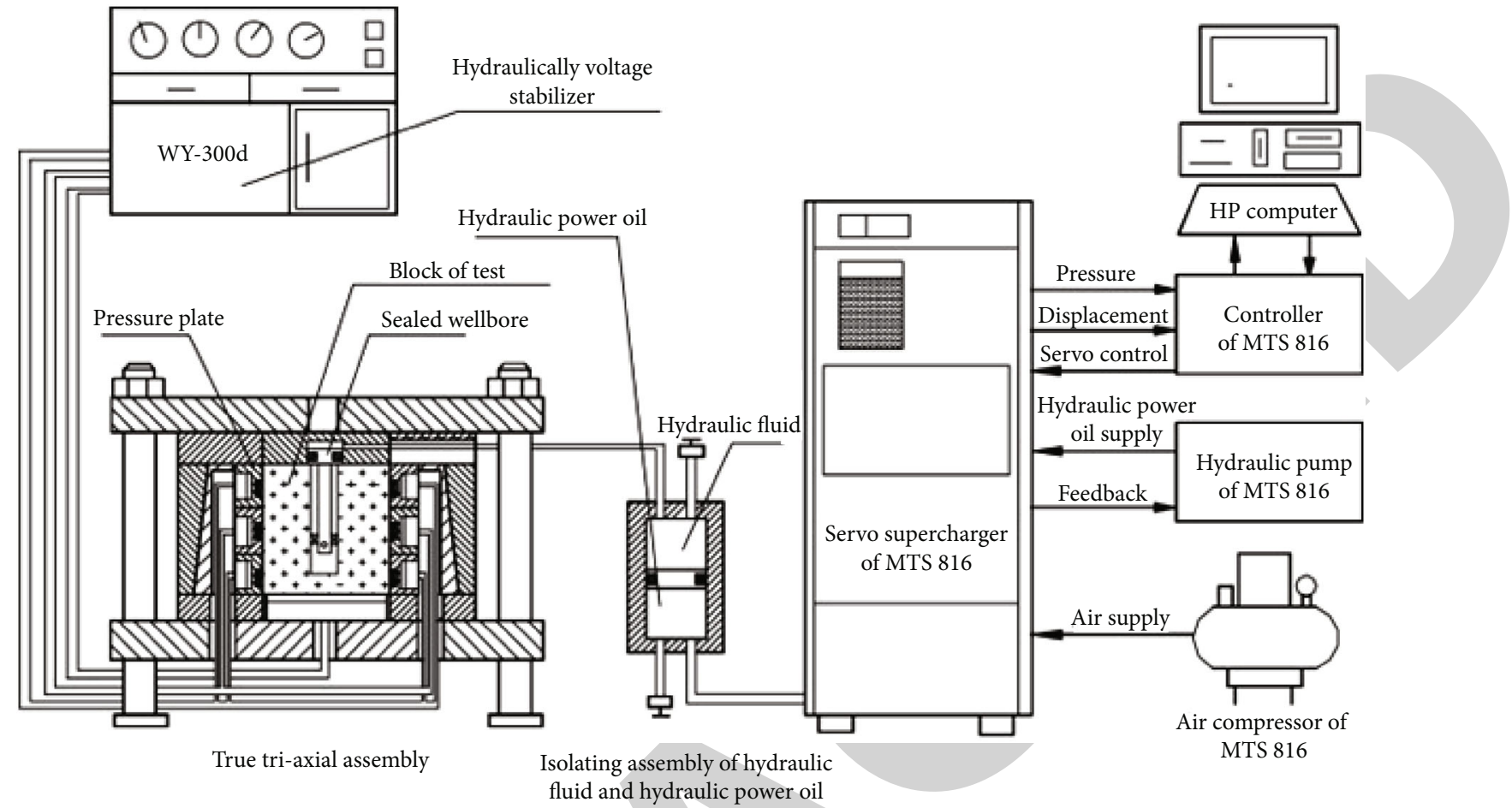

FIgURE 1: Schematic diagram of the triaxial hydraulic fracturing test system.

of $4 \mathrm{~cm}$ in the bottom for fracture initiation. In order to reduce the influence of natural fractures or structural differences in cores on experiments, the cores used in the study all come from the same outcrop. Then, the large outcrop is cut along the bedding direction and perpendicular to the bedding direction. Then, a $20 \mathrm{~mm}$ diameter and $175 \mathrm{~mm}$ length borehole is drilled along the bedding direction to place a wellbore (steel tube) and simulate a horizontal wellbore. All steel pipes are of uniform size: $15 \mathrm{~mm}$ in outer diameter, $8 \mathrm{~mm}$ in inner diameter, and $125 \mathrm{~mm}$ in length. Using high-strength epoxy resin glue to bond steel pipe and borehole wall, a $50 \mathrm{~mm}$ open hole section is reserved at the bottom of the well. Under the action of high-pressure liquid, cracks will occur in the open hole section.

2.2. Experimental Design. Some experiments were designed to understand the effect of changing parameters such as vertical stress difference, flow rate, and fracturing fluid viscosity on the vertical propagation of the hydraulic fracture. In field practice, carbon dioxide fracturing fluid was also an ideal candidate to reduce the harm of fracturing fluid to reservoir formation, especially for the shale gas development. In the experimental design, the influence of carbon dioxide fracturing fluid on fracture propagation was also taken into consideration. The experimental schedule is show in Table 1.

2.3. Experimental Procedures. When the samples were prepared, the cubic model blocks were positioned in between the pressurized pistons in the true triaxial machine. Before the experiment, the samples were measured by the acoustic emission monitoring system and wave velocity data were
TABle 1: Experimental design.

\begin{tabular}{|c|c|c|c|c|c|c|}
\hline \multirow{2}{*}{ Sample } & \multicolumn{3}{|c|}{$\begin{array}{c}\text { In situ } \\
\text { stress }(\mathrm{MPa})\end{array}$} & \multirow{2}{*}{$\begin{array}{c}\text { Stress } \\
\text { difference } \\
(\mathrm{MPa})\end{array}$} & \multirow{2}{*}{$\begin{array}{l}\text { Flow rate } \\
(\mathrm{mL} / \mathrm{min})\end{array}$} & \multirow{2}{*}{$\begin{array}{l}\text { Viscosity } \\
(\mathrm{mPa} \cdot \mathrm{s})\end{array}$} \\
\hline & $\sigma_{\mathrm{v}}$ & $\sigma_{\mathrm{H}}$ & $\sigma_{\mathrm{h}}$ & & & \\
\hline $1 \#$ & 20 & 15 & 10 & 10 & 20 & 45 \\
\hline 2\# & 16 & 15 & 10 & 6 & 20 & 45 \\
\hline 3\# & 16 & 15 & 10 & 6 & 5 & 45 \\
\hline 4\# & 16 & 15 & 10 & 6 & 20 & 2.5 \\
\hline $5 \#$ & 16 & 15 & 10 & 6 & 20 & 0.09 \\
\hline $6 \#$ & 20 & 15 & 10 & 10 & 20 & 0.09 \\
\hline
\end{tabular}

recorded. Then, loading stresses that mimic the in situ stress field were applied to the specimen by using the confining pressure system. When the confining pressure reached a set value, a displacement pump applied the fluid pressure into the open hole section. When the fracturing fluid was injecting, the acoustic emission data was collected at the same time, and the type of acoustic emission event was judged by P-wave. After the experiments, the samples were opened along the fracture surface for observation.

\section{Results and Analysis}

3.1. Vertical Stress Difference. In this section, the vertical stress difference is defined as the difference between the overburden stress $\sigma_{\mathrm{v}}$ and the minimum horizontal principal stress $\sigma_{\mathrm{h}}$. In order to understand the influence of vertical stress, three groups of experiments were designed with the vertical stress difference of $10 \mathrm{MPa}(1 \#)$ and $6 \mathrm{MPa}(2 \#)$. 

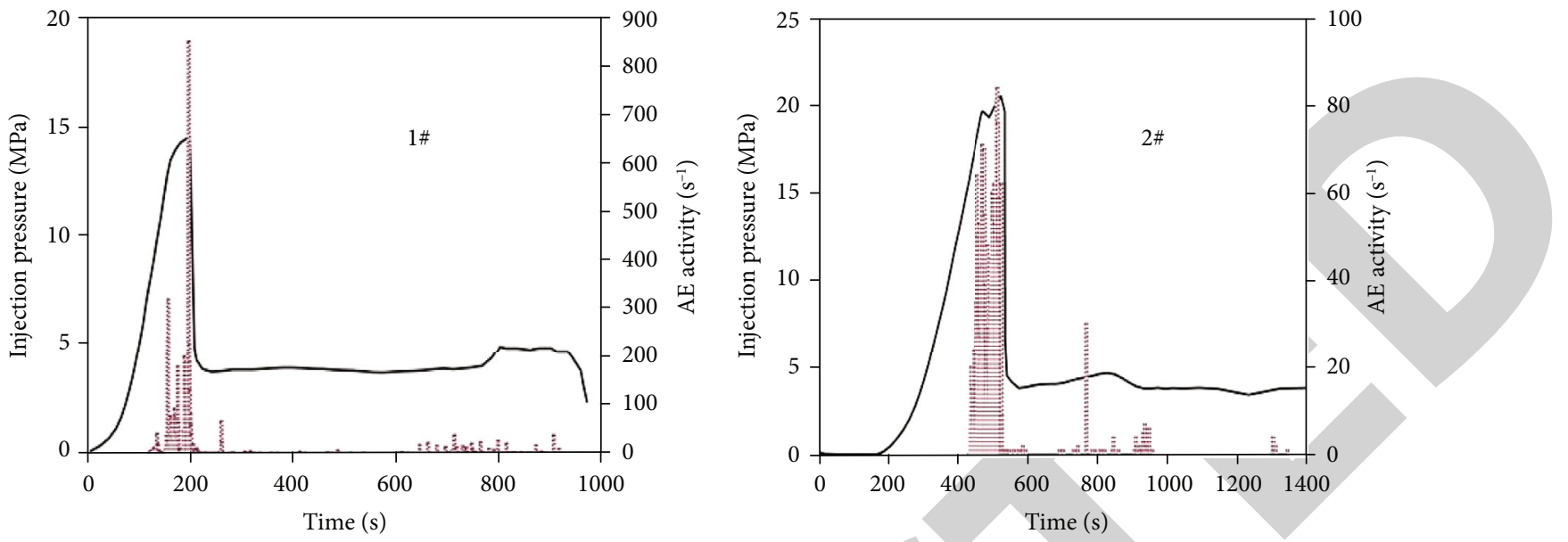

FIGURE 2: The injection pressure curve and $\mathrm{AE}$ activity.
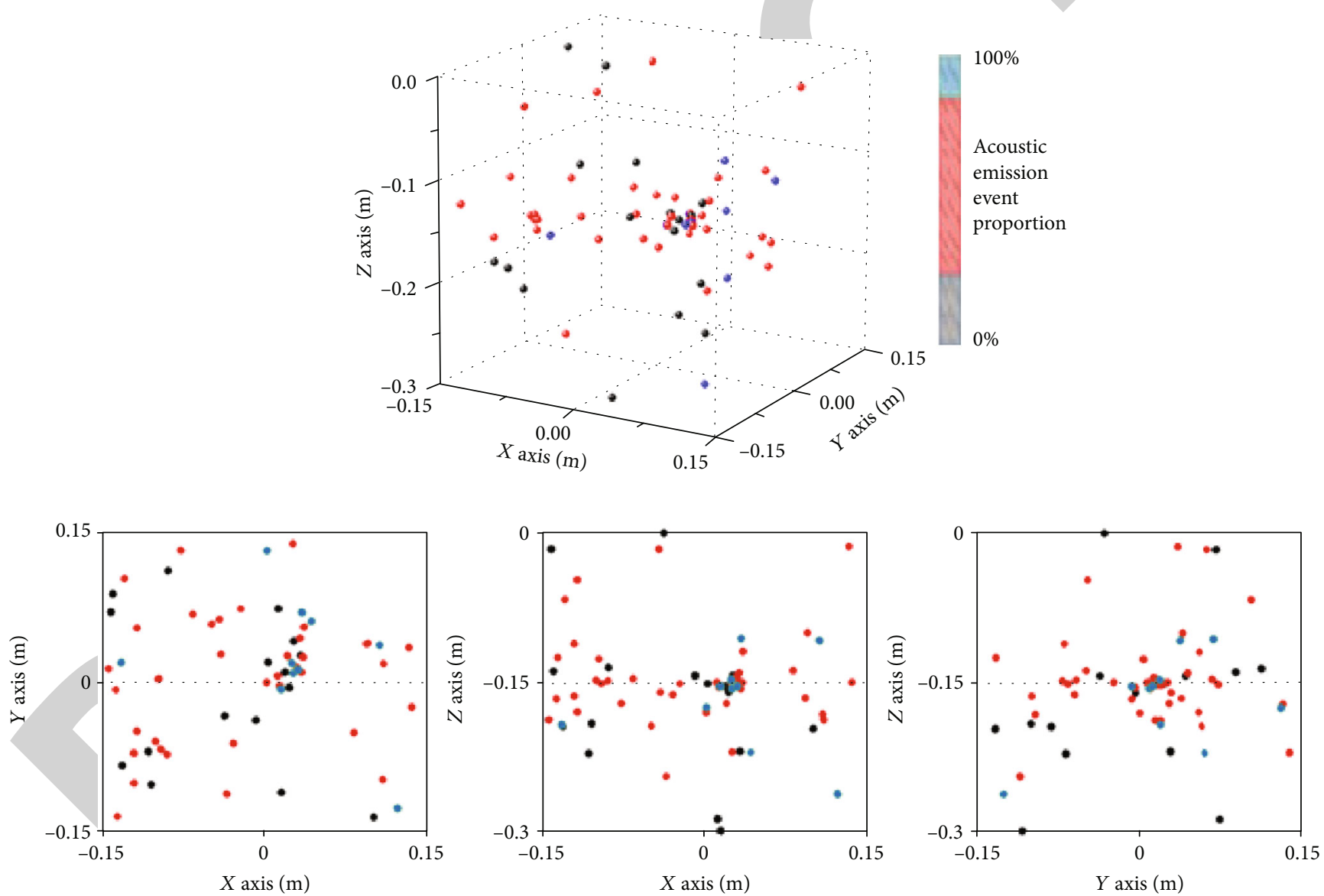

Compression event

Tensile event

Shear event

Figure 3: The AE event distribution of Sample 1\#.

Figure 2 shows the injection pressure of Sample 1\# and Sample 2\#. When the pressure went up to $17.3 \mathrm{MPa}$ (Sample 1\#), it dropped sharply, then stabilized at about $6 \mathrm{MPa}$. In addition, the number of $\mathrm{AE}$ events mainly focused on the initial stage. When the injection pressure stabilized, the $\mathrm{AE}$ events were reduced greatly. When the vertical stress difference was $10 \mathrm{MPa}$ (Sample 1\#), the peak pressure increased slightly from $17.3 \mathrm{MPa}$ to $21.2 \mathrm{MPa}$, then balanced at about $6 \mathrm{MPa}$. What is more, the $\mathrm{AE}$ events increased significantly. 

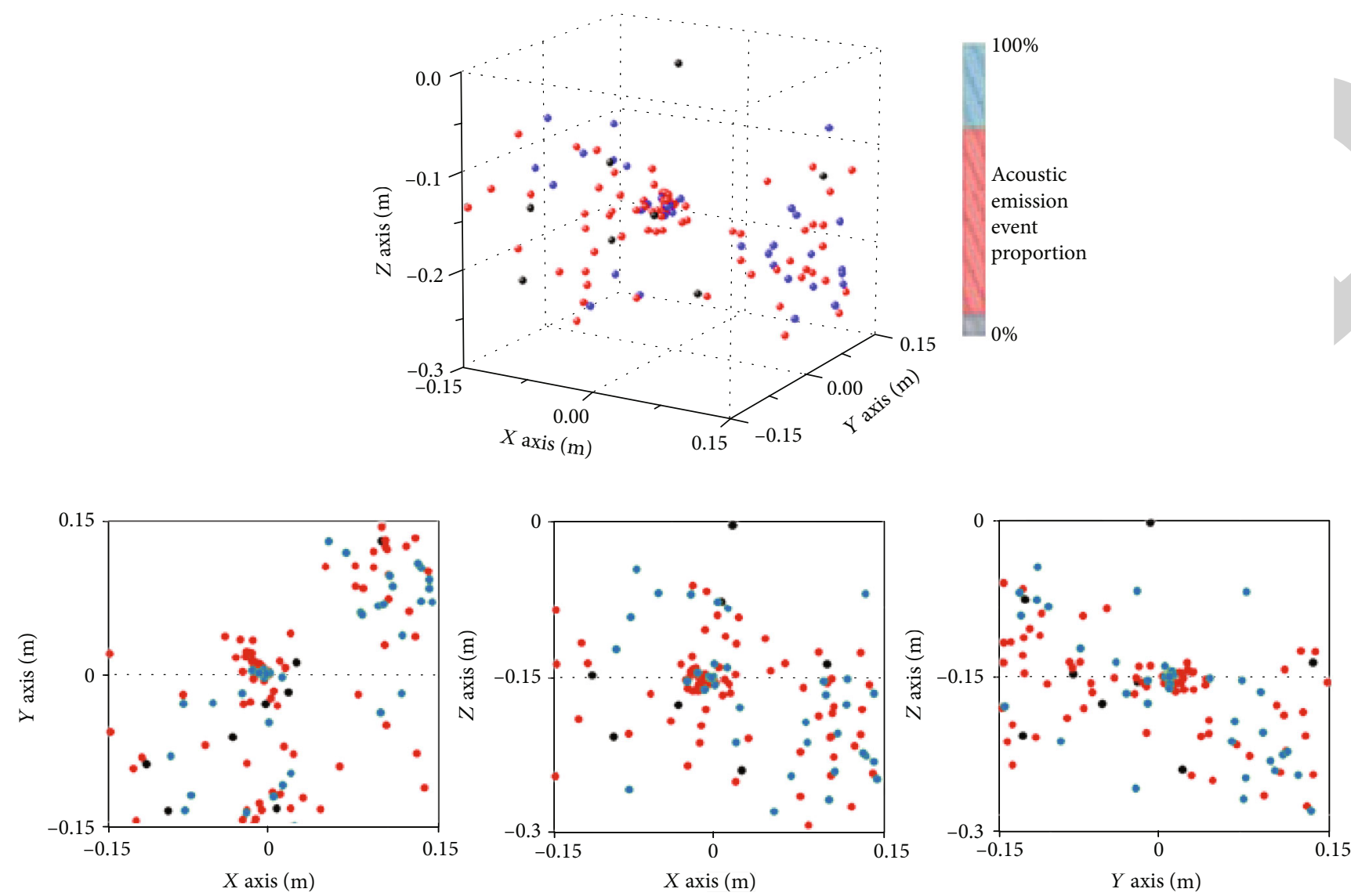

Compression event

- Tensile event

- Shear event

FIgure 4: The AE event distribution of Sample 2\#.

The results of acoustic emission are indicated in Figures 3 and 4. As shown in Figure 3, the AE events mainly distributed near the wellbore, and the shear events were the main events. In addition, the $\mathrm{AE}$ events mainly occurred in the initial injection stage, then dispersedly distributed in the fracture propagation process. For the case that the vertical stress difference was $6 \mathrm{MPa}$ (Figure 4), the number of $\mathrm{AE}$ events increased obviously.

As the sample after fracture demonstrated, a single fracture was created under the high vertical stress difference of $10 \mathrm{MPa}$ (Figure 5(a)); when the vertical stress difference decreased to $6 \mathrm{MPa}$, the hydraulic fracture interacted with bedding planes, and branch fractures were generated (Figure 5(b)). From the experimental results, it can be concluded that with the decrease of vertical stress difference, more branch fractures were created and beddings initiated. In addition, the acoustic emission events demonstrated that the shear events were the main events during the fracture initiation and propagation. The scale of the pictures is $3: 2$.

3.2. The Effect of Flow Rate. Two experiments were carried out to investigate the influence of flow rate on fracture propagation. The injection flow rate of Experiment I (2\#) was
$20 \mathrm{~mL} / \mathrm{min}$, while the injection flow rate of Experiment II (3\#) was $5 \mathrm{~mL} / \mathrm{min}$. When the flow rate was $20 \mathrm{~mL} / \mathrm{min}$ (Figure 6), the number of AE events reduced, which indicated rock failure occurred more frequently under the low flow rate. When the injection pressure climbed to $19.7 \mathrm{MPa}$, it dropped sharply to $6.5 \mathrm{MPa}$; after a period of fluctuation, it stabilized at $6.3 \mathrm{MPa}$. From the comparison of Sample 2\# and Sample 3\#, it can be concluded that the low flow rate can increase the $\mathrm{AE}$ events, which means that the low flow rate is beneficial for the generation of fracture initiation and propagation. As indicated in the distribution of $\mathrm{AE}$ events, the AE events of Sample 3\# mainly occur in the middle of the core, while the AE events of Sample 2\# are scattered throughout the core, which indicates that the low flow rate is more beneficial to create a complex fracture network compared with the high flow rate in shale formation.

As depicted in the interpretation of acoustic emission (Figure 7), the shear event accounted for most acoustic emission events. In addition, the number of acoustic emission above the wellbore plane is significantly higher than below. When the sample was opened along the fracture surface, it was easily found that the hydraulic fractures were cut off by the bedding planes. As demonstrated in Figure 5(c), the two hydraulic fractures in the middle of the sample (3\#) stopped 


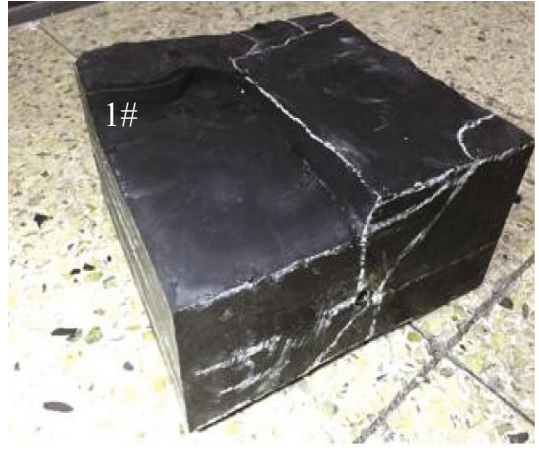

(a)

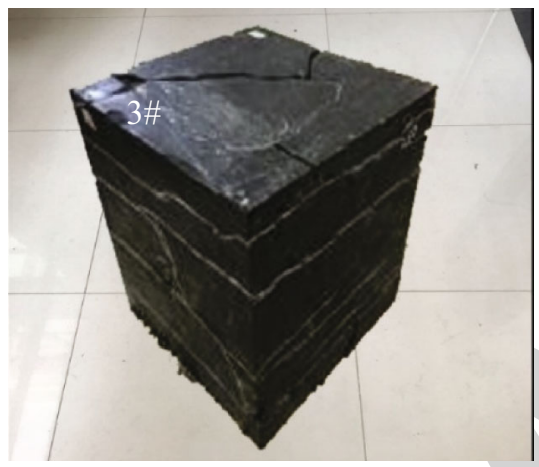

(c)

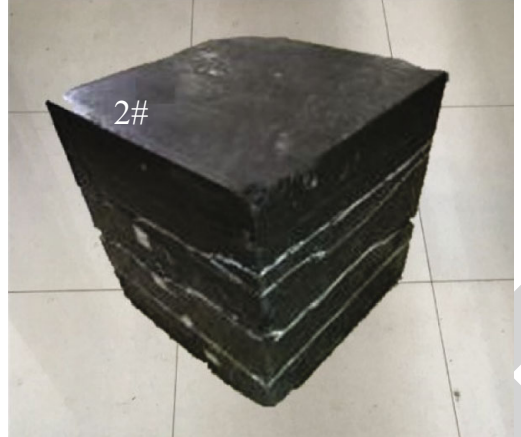

(b)

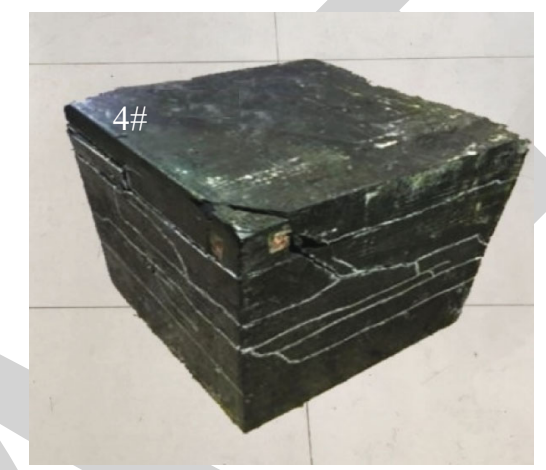

(d)

Figure 5: Fracture geometry after fracturing test.

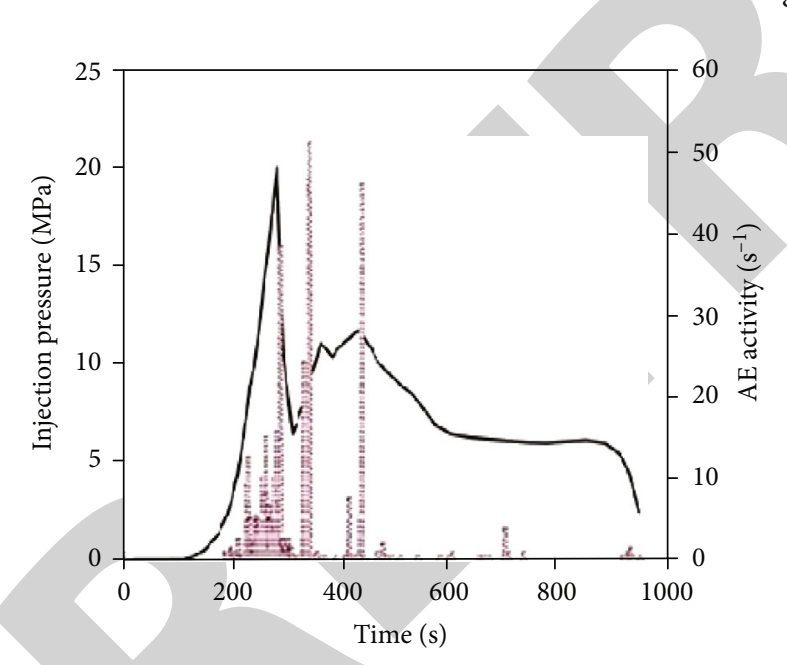

FIGURE 6: The injection pressure curve and AE activity of Sample 3\#.

propagating when they came across the bedding plane. With the increase of the fluid pressure in the fracture, the fracture was initiated in the upper part of the sample. During the propagation process, the hydraulic fractures were cut off several times, according to the injection pressure and the acoustic emission interpretation.

3.3. The Effect of Viscosity. In order to understand the effect of fracturing fluid viscosity on the fracture propagation, the two tests with different fracturing fluid viscosities were conducted.
The fracture fluid viscosity of Experiment I (2\#) was $45 \mathrm{mPa} \bullet \mathrm{s}$, while the fracture fluid viscosity of Experiment II (4\#) was $2.5 \mathrm{mPa} \bullet \mathrm{s}$. As vividly demonstrated in Figure 8, the injection pressure curve experienced several fluctuations, then stabilized at about $6 \mathrm{MPa}$. The fluctuation of the injection pressure curve reflected the hydraulic fracture across the bedding planes. In addition, the acoustic emission events are also accompanied with pressure fluctuations.

The results of interpretation of acoustic emission (Figure 9) indicated that the number of AE events increased greatly compared with Sample 2\#. For the low fracturing fluid viscosity, the $\mathrm{AE}$ events were mainly concentrated in the vicinity of the wellbore; the tensile and shear events were sparse in other parts but almost all over the specimen. As indicated in Figure 5(d), except for the main fracture propagated along the maximum horizontal principle stress, multiple fractures extended along the bedding planes; the complex fracture network was created close to the wellbore. From the experimental results, it can be concluded that the fracturing fluid with high viscosity can reduce the leak-off and shear events, which can reduce the fracture network complexity. The fracturing fluid with low viscosity had higher leak-off coefficient, which can reduce the shear strength of the beddings.

3.4. Supercritical Carbon Dioxide Fracturing. In order to understand the influence of superficial carbon dioxide fracturing fluid on the fracture propagation, two experiments were set to compare the experimental results with linear glue and slick water fracturing fluid (Figure 10): Group I: 

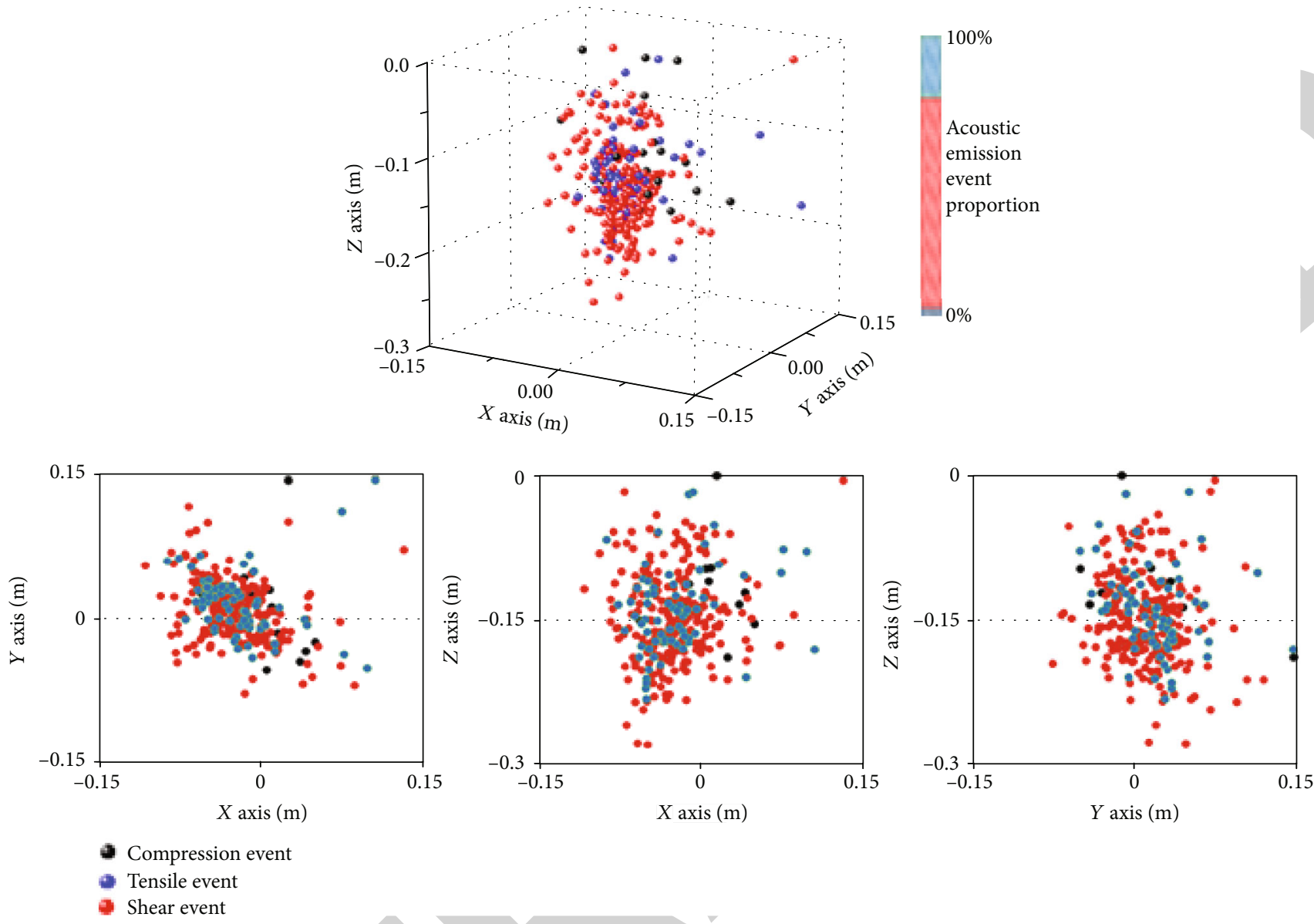

FIgURE 7: The AE event distribution of Sample 3\#.

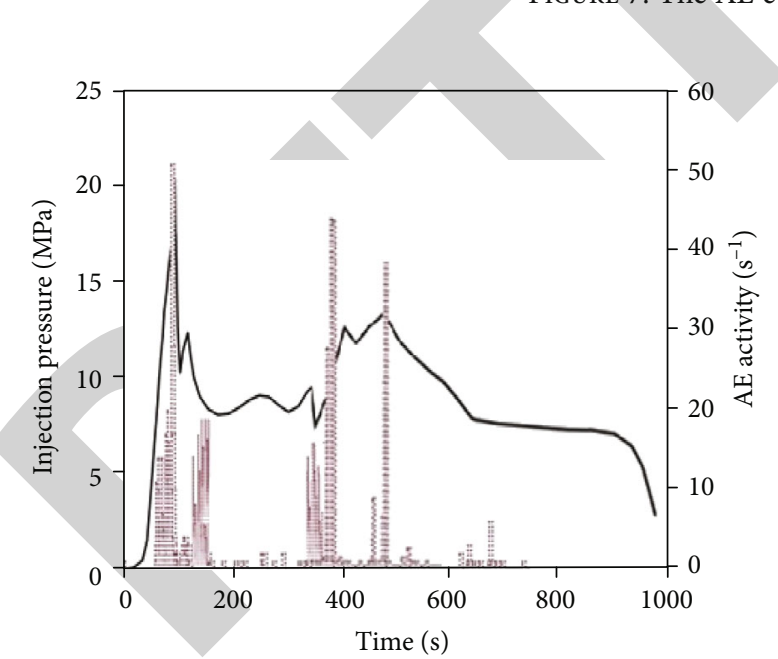

FIGURE 8: The injection pressure curve and AE activity of Sample 4\#.

Specimens 2\# and 5\#; Group II: Specimens 1\# and 6\#. Figures 11 and 12 show the injection pressure curve of Sample 5\# and Sample 6\#. As vividly indicated (Figure 11), the injection pressure of the whole injection process experienced an obvious pressure fluctuation. What is more, the pressure fluctuation is accompanied with the acoustic emission events. Under the low vertical stress difference, the number of acous-

tic emission events increased obviously compared with Specimens 2\# and 5\#. In addition, two pressure peaks appeared during the injection process, and the pressure fluctuation occurred during this period. For Sample 6\# (Figure 12), the frequency of pressure fluctuation was reduced compared with Sample 5\# but was higher than Sample 1\#. Under the high vertical stress difference, the number of AE events was reduced obviously compared with Sample 5\# but was still higher than Sample 1\#.

The interpretation results of acoustic emission (Figures 13 and 14) indicated that the number of $\mathrm{AE}$ events increased dramatically when the superficial carbon dioxide fracturing fluid was adopted in the test. The shear events were the main accidents in the fracturing process (Figure 15). With the vertical stress difference increase, the proportion of tensile event increased. When compared with Samples 2\# and 5\#, the acoustic emission events were more concentrated. Under the high vertical stress difference, the acoustic emission events were relatively dispersed.

\section{Discussion}

From the above experimental results, it can be concluded that the vertical stress difference was the dominant factor affecting the fracture propagation. Under the high vertical stress difference (Figure 16(a)), the injection pressure curve 

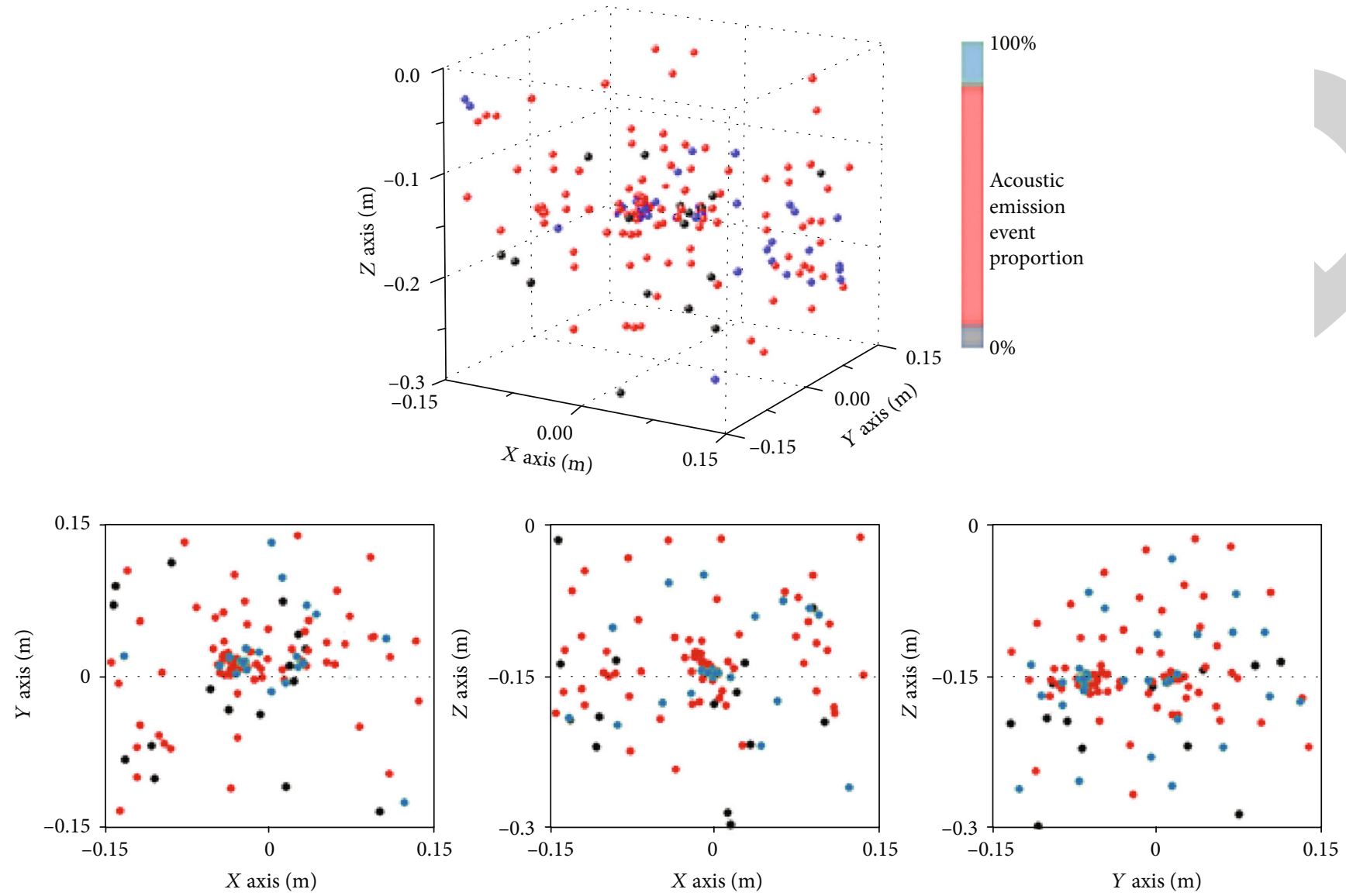

- Compression event

- Tensile event

Shear event

FIGURE 9: The AE event distribution of Sample 4\#.

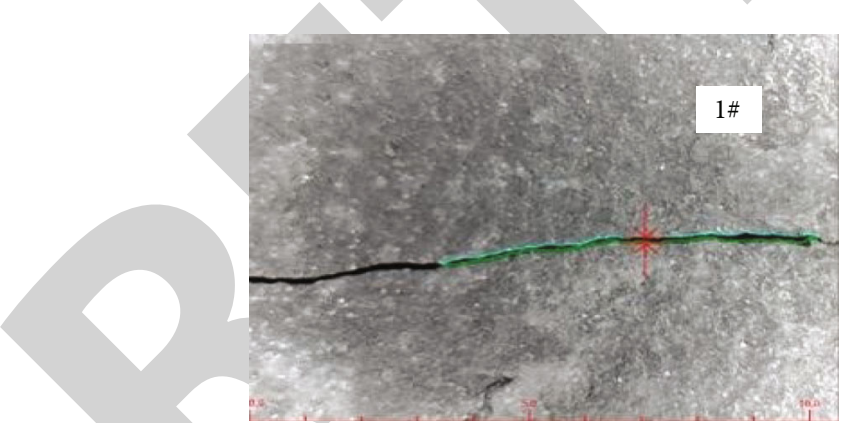

(a)

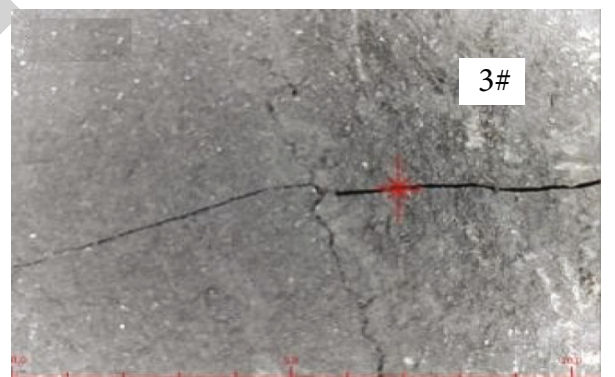

(c)

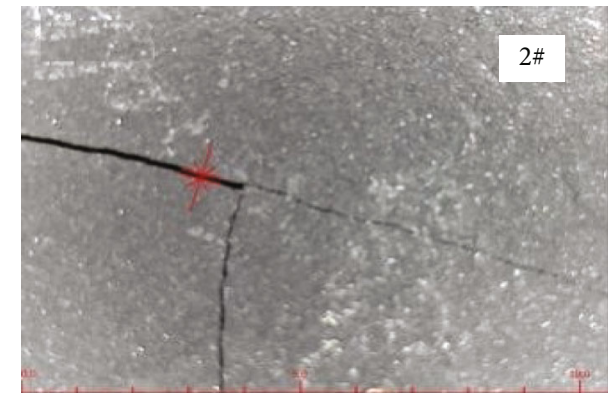

(b)

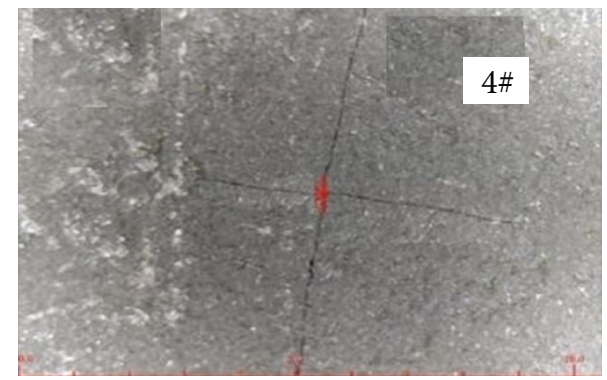

(d)

Figure 10: CT scanning images of hydraulic fracturing. 


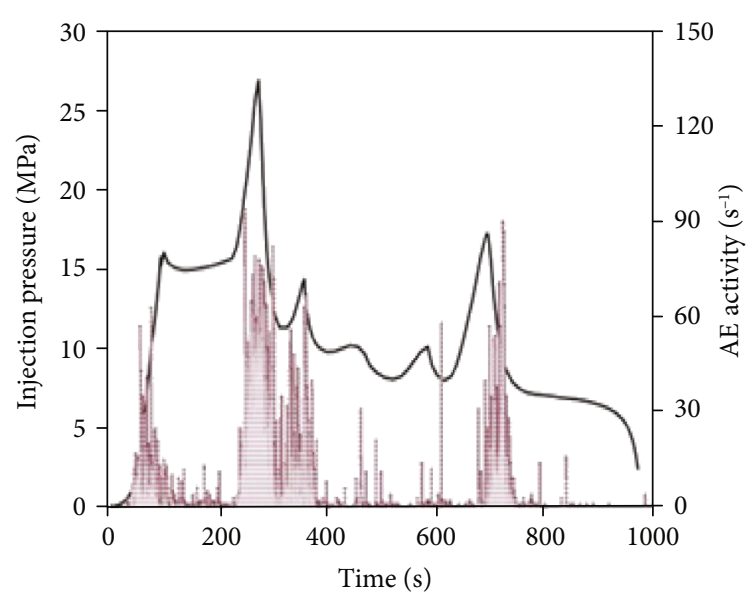

FIGURE 11: The injection pressure curve and AE activity of Sample 5\#.

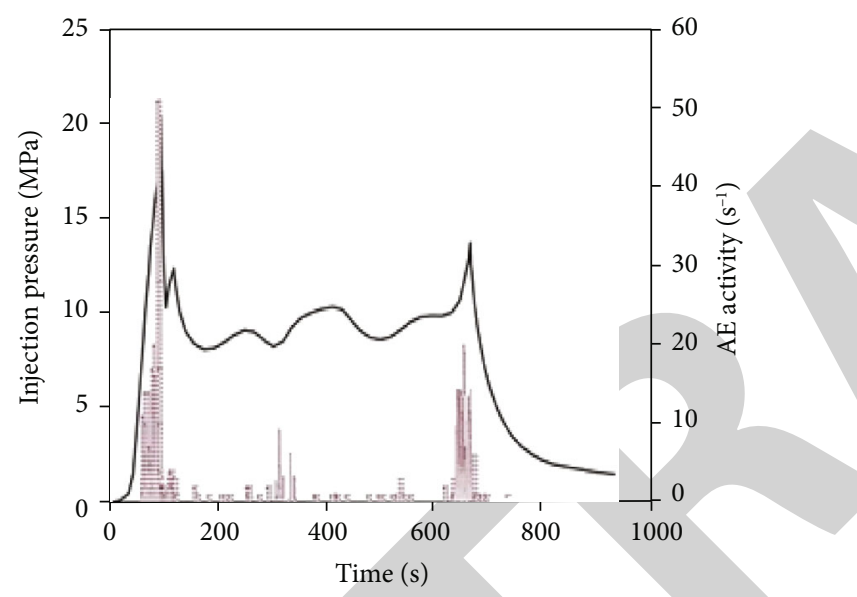

FIgURE 12: The injection pressure curve and AE activity of Sample 6\#.

experienced less pressure fluctuation compared with the low vertical stress difference (Figure 16(b)), which obviously reduced the frequency of acoustic emission events. As shown in Figure 16(a), the bedding was not be activated. From the CT scanning, only a single fracture was created in the test. When the vertical stress difference was $6 \mathrm{MPa}$ (Figure 16(b)), it was obviously seen that the bedding plane was initiated and branch fractures were created. Under the low vertical stress difference, it was easier to generate a complex fracture network.

The viscosity and injection rate of the fracturing fluid are important parameters which affect the generation of a complex fracture network. Under the vertical stress difference of $6 \mathrm{MPa}$, the fracturing fluid with low viscosity and low injection rate could easily leak into the beddings, which could enhance the possibility for interaction between propagators. The flow rate and viscosity were the controllable factors in the hydraulic fracturing field. From the experiments, the high flow rate and viscosity of fracturing fluid were beneficial to generate main fractures which could cross the beddings, while the low injection rate and viscosity of fracturing fluid could accelerate the leak-off of fracturing fluid to bedding planes, which could decrease the shear strength of rocks. According to Figure 10, under the high flow rate and viscosity of fracturing fluid, a smooth hydraulic fracture was created (Figure 10(b)), while the branch fractures were created under the low injection rate (Figure 10(c)); in addition, the hydraulic fracture surface created by the fracturing fluid with low viscosity was much rougher than that of low injection rate and high viscosity. As demonstrated in Figures 16(c) and $16(\mathrm{~d})$, the hydraulic fractures were more likely to deviate due to the reduction of shear strength.

Supercritical carbon dioxide fracturing was an innovative method applied in the fracturing practice. From the experiments, the fracture geometry under supercritical carbon dioxide fracturing was much more complex compared with that under hydraulic fracturing. Under the low vertical stress difference (Figure 17(a)), more branch fractures were created under the vertical stress $6 \mathrm{MPa}$. From the CT scanning, the fracture network (Figure 18(a)) was much more complicated than that of $10 \mathrm{MPa}$. When the vertical stress difference was $10 \mathrm{MPa}$ (Figure 18(b)), the fracture geometry was relatively simple, but the branch fractures could be seen clearly. The CT scanning also verified the result. Comparing Sample 1\# with Sample 6\#, Sample 2\# with Sample\# 5, it is found that supercritical carbon dioxide can form a more complex fracture network when compared with waterbased fracturing fluid. The main reason is that the viscosity of supercritical carbon dioxide is much less than that of water-based fracturing fluid, and the low viscosity of fracturing fluid is beneficial for the generation of a complex fracture network.

In addition, the proportion of the AE events also indicates that the shear failure is the main failure mode in shale formation. According to the $\mathrm{AE}$ event distribution in the previous section, it can be found that the shear events of Sample 1\#, Sample 2\#, Sample 3\#, Sample 4, and Sample 5 are $65.8 \%, 67.2 \%, 77.4 \%, 82.1 \%$, and $82.3 \%$, while the tensile failure are $11.3 \%, 16.8 \%, 19.5 \%$, $12.6 \%$, and 11.2. It demonstrates that the shear failure is much easier to occur in formation.

\section{Conclusion}

The laboratory experiments are conducted to investigate the factors influencing the vertical extension of hydraulic fractures. In addition, the advantages of supercritical carbon dioxide fracturing are studied in this research. The main conclusions are listed as follows:

(1) Under high vertical stress, the main fractures are more likely to cross the bedding plane; a single fracture is created. The hydraulic fractures deviate to the beddings under the low vertical stress difference, which is beneficial to form a complex fracture network

(2) High injection rate and viscosity of fracturing fluid can help create the main fracture due to the less leak-off of the fracturing fluid, while more branch fractures are created under the low injection rate and viscosity of 

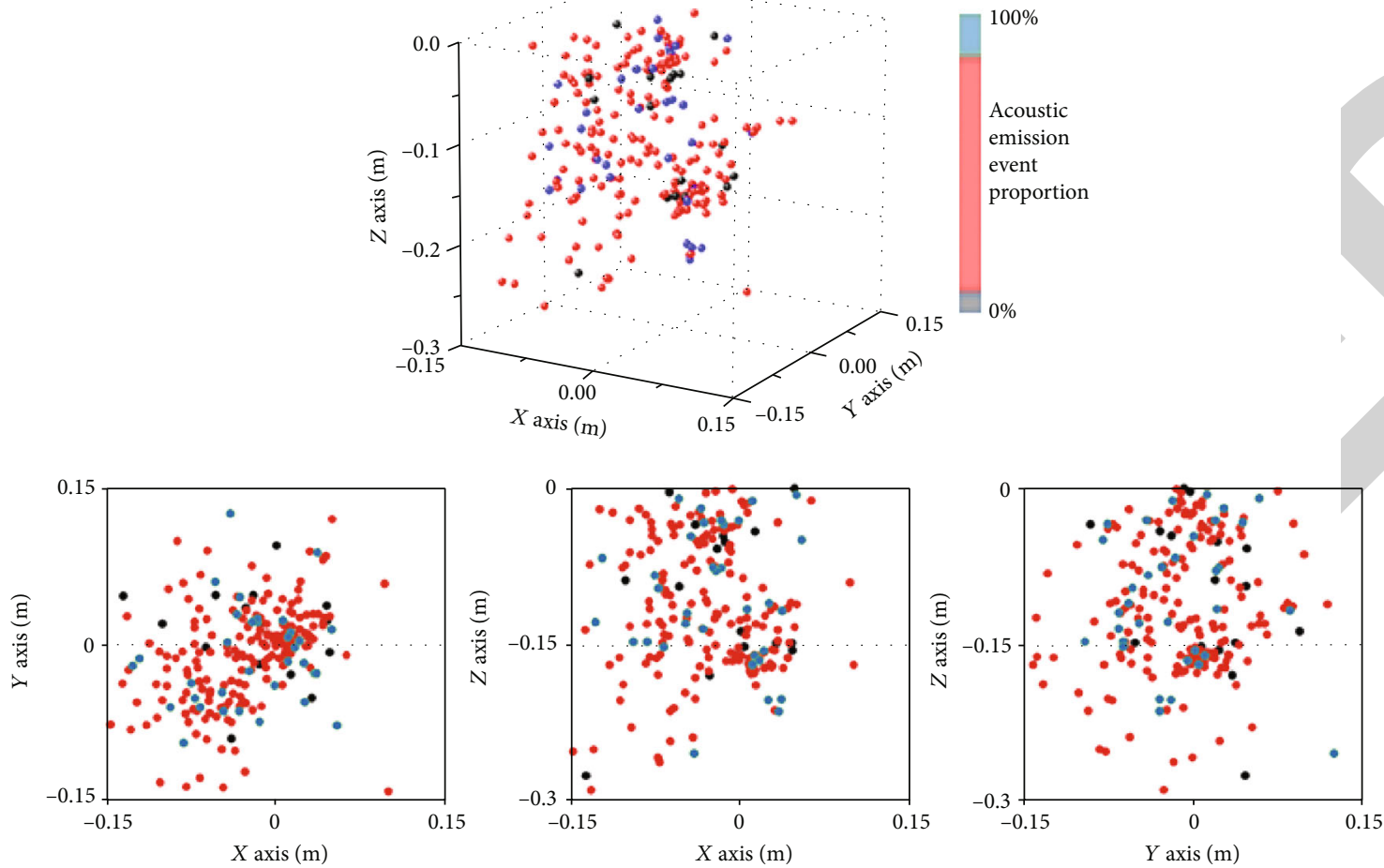

- Compression event

- Tensile event

- Shear event

Figure 13: The AE event distribution of Sample 5\#.
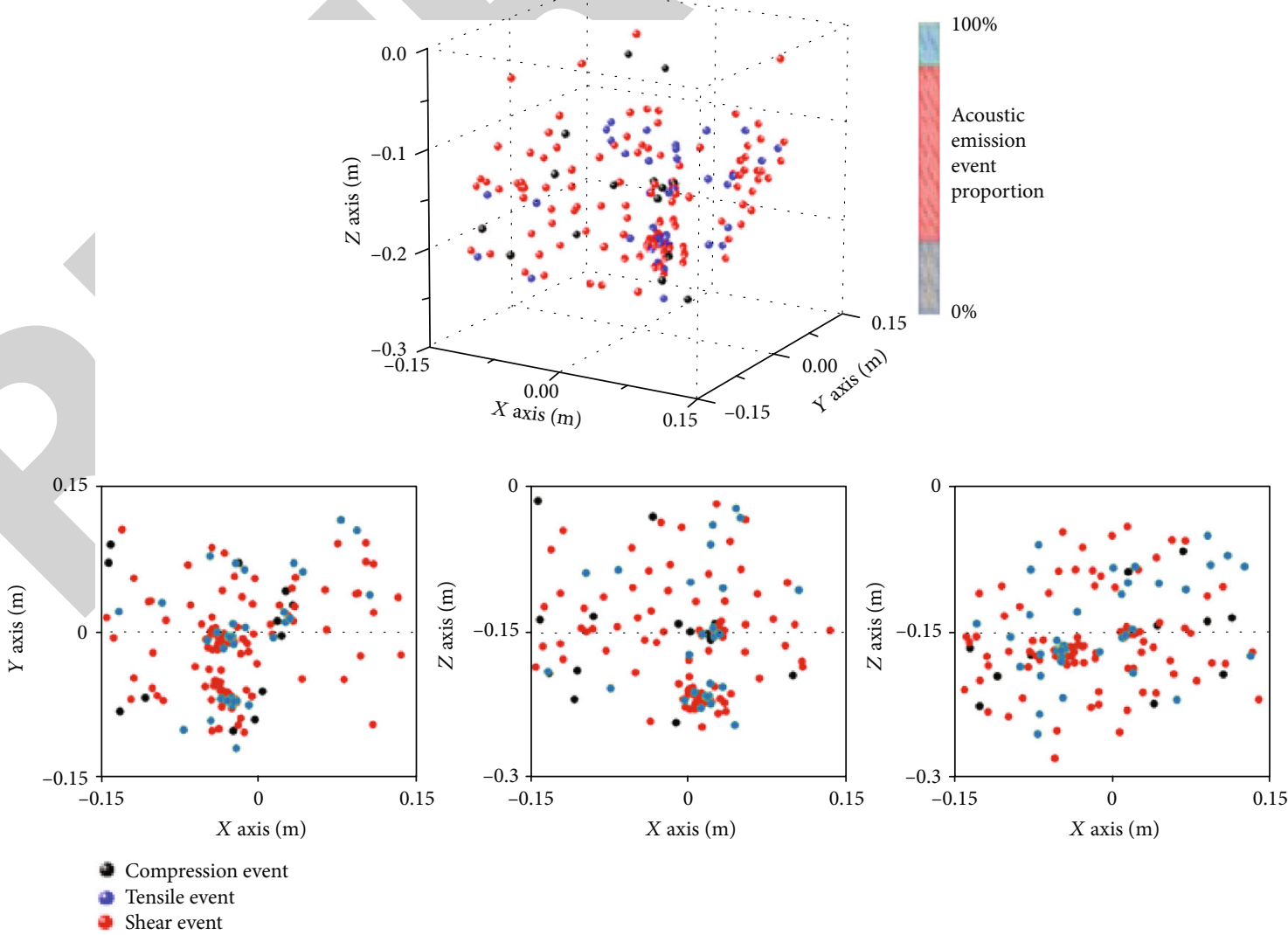

FIgURE 14: The AE event distribution of Sample 6\#. 

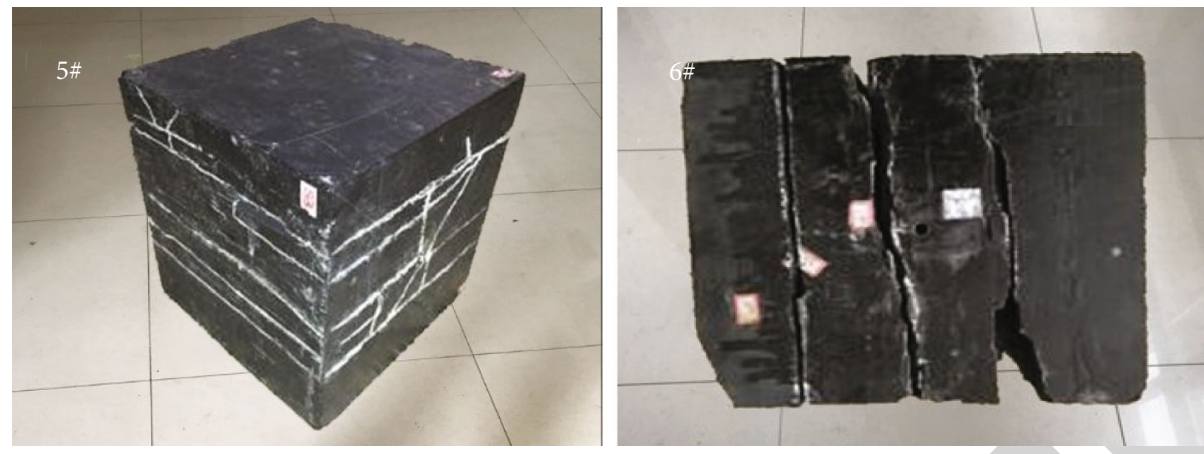

FIGURE 15: Fracture geometry of after fracturing test superficial carbon dioxide fracturing.
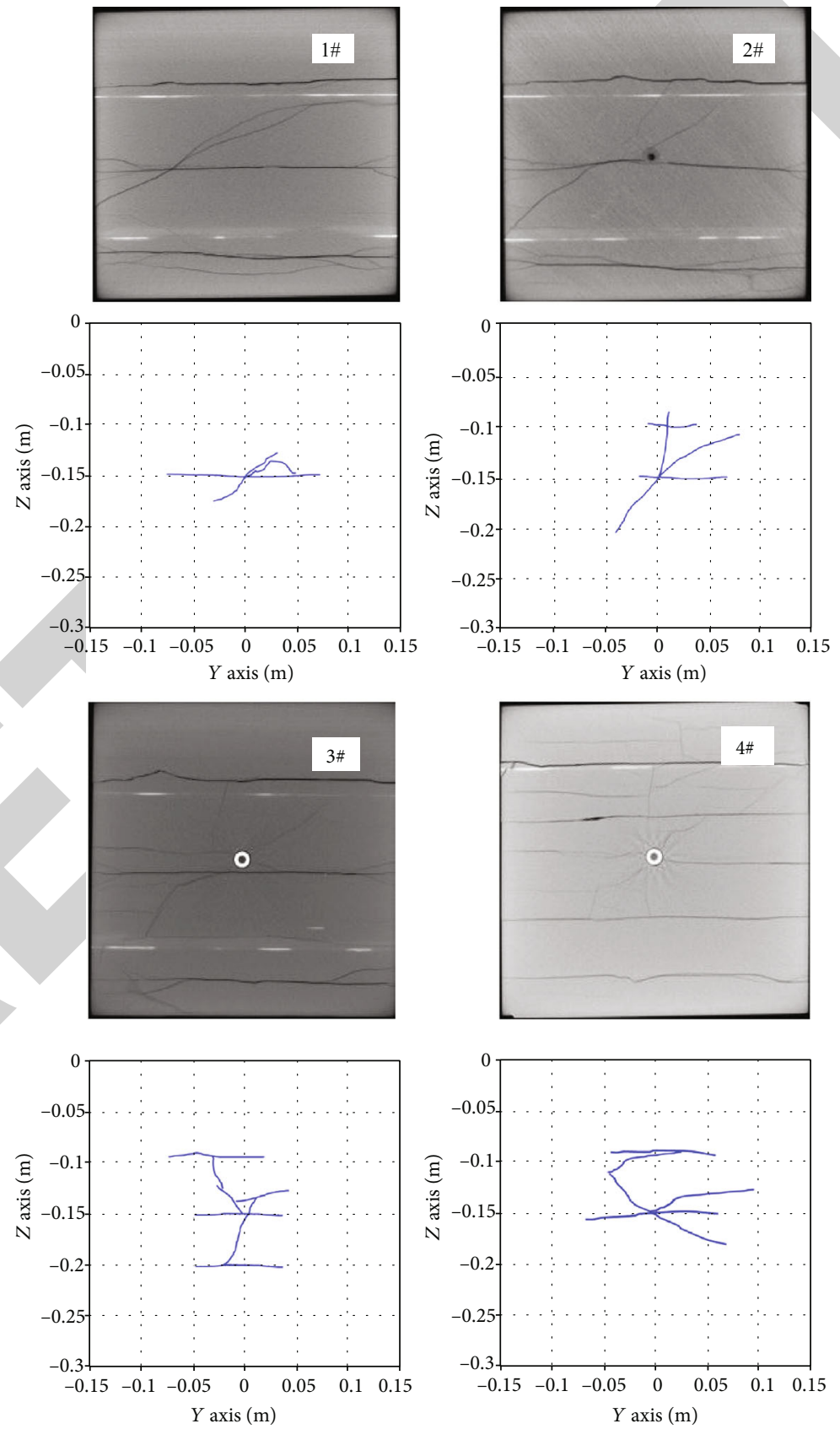

FIGURE 16: The explanation results of CT scanning. 

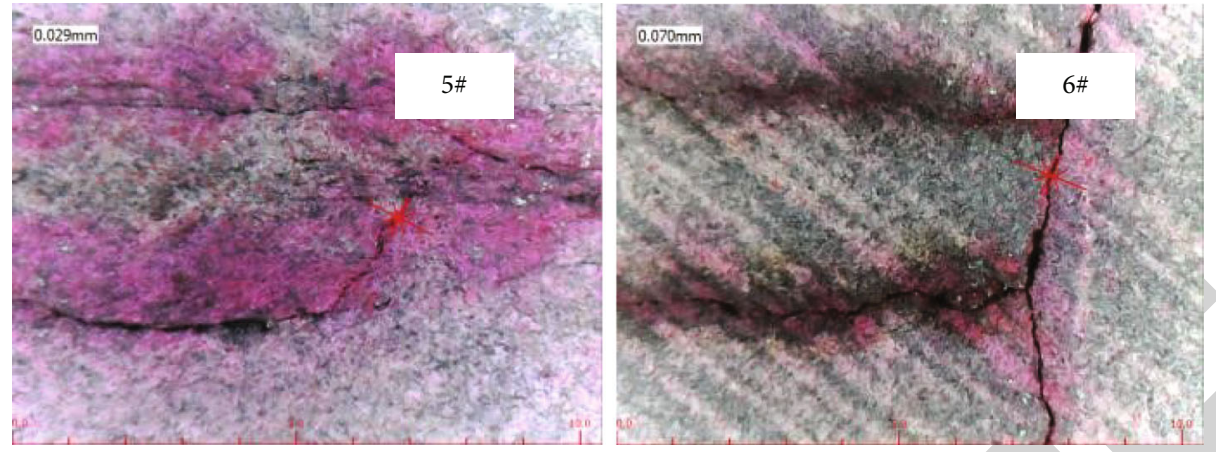

FIGURE 17: CT scanning images of superficial carbon dioxide fracturing.
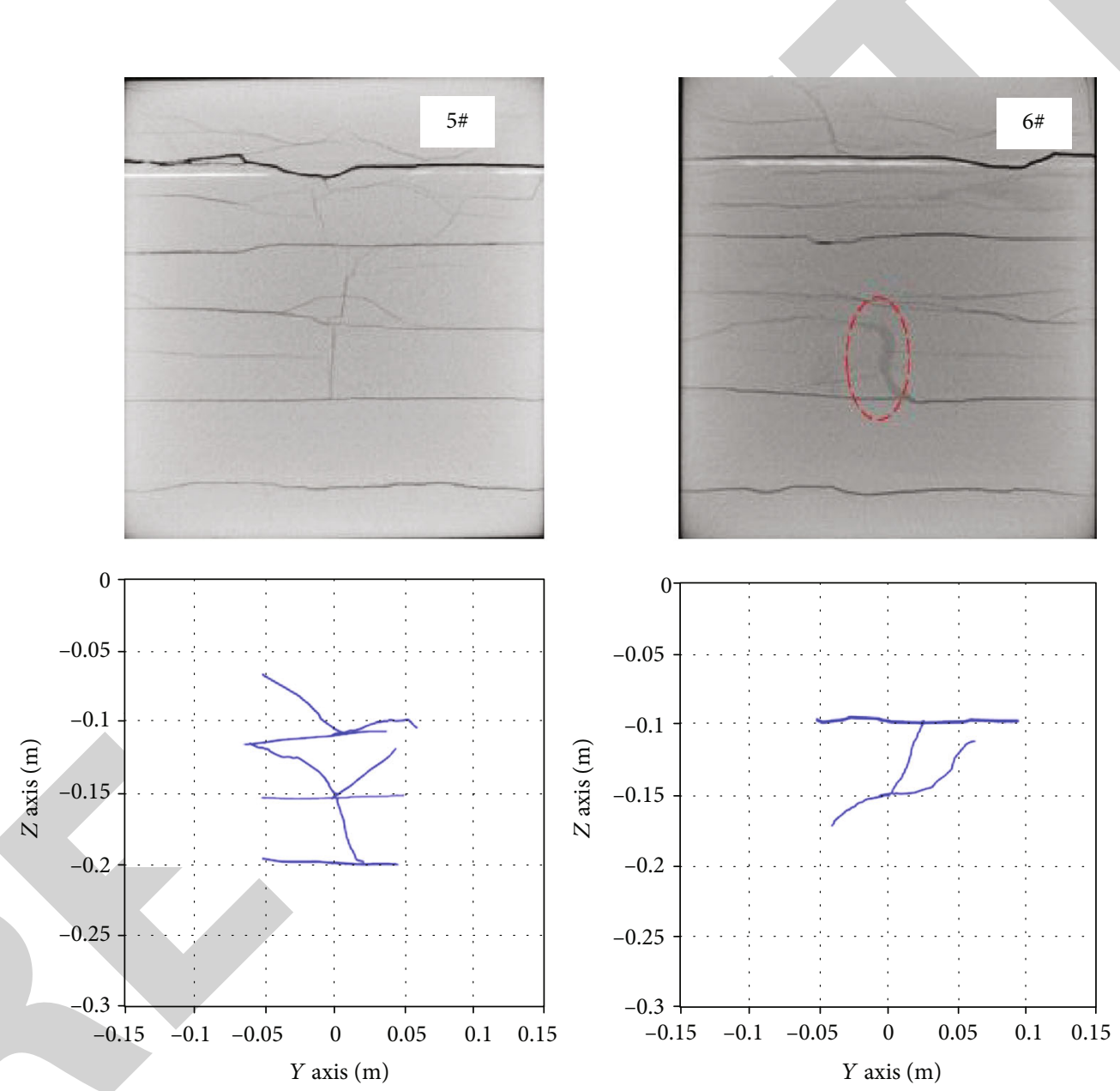

FIGURE 18: The explanation results of CT scanning.

fracturing fluid. The influence of the flow rate and viscosity of the fracturing fluid on the vertical propagation of hydraulic fractures can be considered a combined effect. In the stimulation of shale reservoir, a mixed fracturing method is proposed that high viscosity is used to create a straight main fracture near the wellbore, then altered to a low viscous fluid combined with a low injection rate to generate a complex fracture network in the distance
(3) Acoustic emission events accompany the fluctuation of injection pressure. During the initiation and extension of hydraulic fractures, the injection curve experiences a severe fluctuation, and the acoustic emission rate is relatively high; when the injection curve stabilized, the acoustic emission rate is relatively low. In addition, the shear events are the main acoustic emission events in the hydraulic fracturing process 
(4) There is a good spatial correspondence between AE events and fracture morphology. The hydraulic fracture was initiated and propagated from the open hole section, and the number of acoustic emission events is more and distributed densely. The more the acoustic emission events are, the more fractures are created

(5) Compared with the hydraulic fracturing, the supercritical carbon dioxide fracturing has an obvious advantage for creating a complex fracture network, especially for the high vertical stress difference formation

\section{Data Availability}

The data used to support the findings of this study are included within the article.

\section{Conflicts of Interest}

The authors declare that they have no conflicts of interest.

\section{Acknowledgments}

This study was financially supported by the National Natural Science Foundation of China (41688103), the Shandong Provincial Natural Science Foundation, China (ZR2017PD001, ZR2018MD010), the Science Foundation of Chinese Academy of Geological Sciences (JYYWF20181201), the Shandong Provincial Key Laboratory of Depositional Mineralization \& Sedimentary Minerals, Shandong University of Science and Technology, China (DMSM2017018), and the 2017 Key Research and Development Plan Project of Shandong Province (2017CXGC1602).

\section{References}

[1] D. G. Crosby, Z. Yang, and S. S. Rahman, "Methodology to predict the initiation of multiple transverse fractures from horizontal wellbores," Journal of Canadian Petroleum Technology, vol. 40, no. 10, 2001.

[2] A. Turon, P. P. Camanho, J. Costa, and C. G. Dávila, "A damage model for the simulation of delamination in advanced composites under variable-mode loading," Mechanics of Materials, vol. 38, no. 11, pp. 1072-1089, 2006.

[3] C. Yan and Y.-Y. Jiao, "A 2D fully coupled hydro-mechanical finite-discrete element model with real pore seepage for simulating the deformation and fracture of porous medium driven by fluid," Computers \& Structures, vol. 196, pp. 311-326, 2018.

[4] C. Yan, Y.-Y. Jiao, and H. Zheng, "A fully coupled threedimensional hydro-mechanical finite discrete element approach with real porous seepage for simulating 3D hydraulic fracturing," Computers and Geotechnics, vol. 96, pp. 73-89, 2018.

[5] C. Yan and H. Zheng, "A two-dimensional coupled hydromechanical finite-discrete model considering porous media flow for simulating hydraulic fracturing," International Journal of Rock Mechanics and Mining Sciences, vol. 88, pp. 115128, 2016.

[6] C. Yan and H. Zheng, “Three-dimensional hydromechanical model of hydraulic fracturing with arbitrarily discrete frac- ture networks using finite-discrete element method," International Journal of Geomechanics, vol. 17, no. 6, p. 04016133, 2017.

[7] R. Yuan, L. Jin, C. Zhu, S. Vitthal, and B. Xu, "High pressure stimulation - impact of hydraulic fracture geometry to unconventional gas appraisal and development in compressional settings," in SPE Annual Technical Conference and Exhibition New Orleans, Society of Petroleum Engineers, Louisiana, USA, 2013.

[8] G. M. Zhang, H. Liu, J. Zhang, H. A. Wu, and X. X. Wang, "Three-dimensional finite element simulation and parametric study for horizontal well hydraulic fracture," Journal of Petroleum Science and Engineering, vol. 72, no. 3-4, pp. 310-317, 2010.

[9] C. H. Wu, S. S. Yi, and M. M. Sharma, "Proppant distribution among multiple perforation clusters in a horizontal wellbore," in SPE Hydraulic Fracturing Technology Conference and Exhibition, Society of Petroleum Engineers, 2017.

[10] K. Wu, J. E. Olson, and M. T. Balhoff, "Study of Multiple Fracture Interaction Based on An Efficient Three-Dimensional Displacement Discontinuity Method," in 49th US Rock Mechanics/Geomechanics Symposium, American Rock Mechanics Association, 2015.

[11] X. Weng, O. Kresse, C. E. Cohen, R. Wu, and H. Gu, "Modeling of hydraulic fracture network propagation in a naturally fractured formation," in SPE Hydraulic Fracturing Technology Conference, Society of Petroleum Engineers, 2011.

[12] L. Xie, K.-B. Min, and B. Shen, "Simulation of hydraulic fracturing and its interactions with a pre-existing fracture using displacement discontinuity method," Journal of Natural Gas Science and Engineering, vol. 36, pp. 1284-1294, 2016.

[13] C. Yan, H. Zheng, G. Sun, and X. Ge, "Combined finitediscrete element method for simulation of hydraulic fracturing," Rock Mechanics and Rock Engineering, vol. 49, no. 4, pp. 1389-1410, 2016.

[14] J. Guo, Q. Lu, H. Zhu, Y. Wang, and L. Ma, "Perforating cluster space optimization method of horizontal well multi-stage fracturing in extremely thick unconventional gas reservoir," Journal of Natural Gas Science and Engineering, vol. 26, pp. 16481662, 2015.

[15] J. Guo, X. Zhao, H. Zhu, X. Zhang, and R. Pan, "Numerical simulation of interaction of hydraulic fracture and natural fracture based on the cohesive zone finite element method," Journal of Natural Gas Science and Engineering, vol. 25, pp. 180-188, 2015.

[16] C. Yan and H. Zheng, "FDEM-flow3D: a 3D hydromechanical coupled model considering the pore seepage of rock matrix for simulating three-dimensional hydraulic fracturing," Computers and Geotechnics, vol. 81, pp. 212-228, 2017.

[17] D. A. Chuprakov, A. Akulich, E. Siebrits, and M. J. Thiercelin, "Hydraulic fracture propagation in a naturally fractured reservoir," in SPE Oil and Gas India Conference and Exhibition Mumbai, Society of Petroleum Engineers, India, 2010.

[18] J. Fan, J. Chen, D. Jiang, S. Ren, and J. Wu, "Fatigue properties of rock salt subjected to interval cyclic pressure," International Journal of Fatigue, vol. 90, pp. 109-115, 2016.

[19] E. Gordeliy and A. Peirce, "Coupling schemes for modeling hydraulic fracture propagation using the XFEM," Computer Methods in Applied Mechanics and Engineering, vol. 253, pp. 305-322, 2013. 
[20] Z. Liu, M. Chen, and G. Zhang, "Analysis of the influence of a natural fracture network on hydraulic fracture propagation in carbonate formations," Rock Mechanics and Rock Engineering, vol. 47, no. 2, pp. 575-587, 2014.

[21] B. Hou, M. Chen, W. Cheng, and C. Diao, "Investigation of hydraulic fracture networks in shale gas reservoirs with random fractures," Arabian Journal for Science and Engineering, vol. 41, no. 7, pp. 2681-2691, 2016.

[22] J. Zhou, M. Chen, Y. Jin, and G. Q. Zhang, "Analysis of fracture propagation behavior and fracture geometry using a triaxial fracturing system in naturally fractured reservoirs," International Journal of Rock Mechanics and Mining Sciences, vol. 45, no. 7, pp. 1143-1152, 2008.

[23] H. P. Lee, J. E. Olson, J. Holder, J. F. W. Gale, and R. D. Myers, "The interaction of propagating opening mode fractures with preexisting discontinuities in shale," Journal of Geophysical Research: Solid Earth, vol. 120, no. 1, pp. 169-181, 2015.

[24] R. Keshavarzi and S. Mohammadi, "A new approach for numerical modeling of hydraulic fracture propagation in naturally fractured reservoirs," in SPE/EAGE European Unconventional Resources Conference and Exhibition Vienna, Society of Petroleum Engineers, Austria, 2012.

[25] X. Jin, S. N. Shah, J. C. Roegiers, and B. Zhang, "An integrated petrophysics and geomechanics approach for fracability evaluation in shale reservoirs," SPE Journal, vol. 20, no. 03, pp. 518526, 2015.

[26] X. Jin, S. N. Shah, J. A. Truax, and J.-C. Roegiers, "A practical petrophysical approach for brittleness prediction from porosity and sonic logging in shale reservoirs," in SPE Annual Technical Conference and Exhibition Amsterdam, Society of Petroleum Engineers, The Netherlands, 2014. 